\title{
La prétérition comme outil de stratégie rhétorique
}

Manoeuvring strategically with praeteritio

\section{Francisca Snoeck Henkemans}

Traducteur : Sivan Cohen-Wiesenfeld

\section{(2) OpenEdition \\ Journals}

Édition électronique

URL : http://journals.openedition.org/aad/217

DOI : $10.4000 /$ aad. 217

ISSN : 1565-8961

\section{Éditeur}

Université de Tel-Aviv

\section{Référence électronique}

Francisca Snoeck Henkemans, "La prétérition comme outil de stratégie rhétorique », Argumentation et Analyse du Discours [En ligne], 2 | 2009, mis en ligne le 01 avril 2009, consulté le 23 septembre 2019. URL : http://journals.openedition.org/aad/217 ; DOI : 10.4000/aad.217

\section{Ce document a été généré automatiquement le 23 septembre 2019}

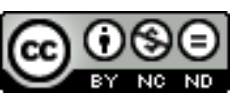

Argumentation \& analyse du discours est mis à disposition selon les termes de la licence Creative Commons Attribution - Pas d'Utilisation Commerciale - Pas de Modification 4.0 International. 


\title{
La prétérition comme outil de stratégie rhétorique
}

\author{
Manoeuvring strategically with praeteritio
}

Francisca Snoeck Henkemans

Traduction : Sivan Cohen-Wiesenfeld

\section{NOTE DE L'ÉDITEUR}

Ce texte est la version française d'un article publié dans Argumentation 2009 : 2, « Perelman and Beyond. Current Issues in Argumentation Studies ", Amossy, Koren and Yanoshevsky (eds). Traduit de l'anglais par Sivan Cohen-Wiesenfeld.

\section{Introduction}

1 Dans cet article, la prétérition sera étudiée à l'intérieur du cadre théorique développé au cours de ces dix dernières années par Frans van Eemeren et Peter Houtlosser, qui associent des éléments de rhétorique à une approche pragma-dialectique de l'argumentation. Pour van Eemeren et Houtlosser, la pratique argumentative ordinaire possède une double dimension, dialectique et rhétorique (2002:9). Les argumentateurs n'ont généralement pas pour seul but de faire triompher une position, mais également de tenir un discours raisonnable, c'est-à-dire en accord avec les règles de la discussion critique (2002: 135). Selon eux, il n'y a aucune raison de supposer que les normes rhétoriques de persuasion soient nécessairement en contradiction avec la notion critique de raisonnable. Il existe cependant une tension potentielle dans le fait de poursuivre un but à la fois dialectique et rhétorique (2002: 135). Pour réduire cette discordance, les argumentateurs emploient ce que van Eemeren et Houtlosser nomment un processus d'« ajustement stratégique ». Celui-ci comporte trois aspects ${ }^{1}$ :

L'ajustement stratégique peut consister dans un choix opportun parmi les éléments $\mathrm{du}$ «potentiel argumentatif » associé à une étape particulière de la discussion, ou 
dans celui d'une réponse adaptée aux « exigences de l'auditoire », ou encore dans l'exploitation des « dispositifs de présentation » appropriés. Les deux parties sont supposées sélectionner les éléments qu'elles savent le mieux manipuler, ou qui soutiennent le mieux leur position, développer les aspects les plus acceptables pour leur auditoire et présenter leur argumentation de la façon la plus efficace (van Eemeren et Houtlosser $2002: 139$ ).

2 L'ajustement stratégique peut « déraper » et devenir fallacieux dans le cas où l'une des parties laisse ses buts rhétoriques prendre le dessus sur ses objectifs dialectiques, et est amenée à violer une ou plusieurs règles de la discussion critique.

Dans mes travaux récents, je recherche les utilisations possibles de dispositifs de présentation spécifiques dans des buts d'ajustement stratégique ${ }^{2}$. Pour ce faire, j'examine le rôle que peuvent jouer des moyens stylistiques comme la métonymie, les questions rhétoriques et la prétérition dans la présentation efficace des choix discursifs de l'argumentateur, à chaque étape de la discussion. L'approche adoptée dans le cadre de cette recherche est la suivante: une analyse des effets interactionnels et communicationnels, selon la stylistique classique et moderne, de ces tropes et figures de pensée est tout d'abord présentée afin d'obtenir un meilleur aperçu de leur potentiel stratégique; ces effets sont ensuite analysés dans la perspective du processus d'ajustement stratégique.

4 Le présent article traite de la prétérition, figure de pensée par laquelle, selon le Oxford Dictionary of English (2005), « on attire l'attention sur une chose par le fait d'annoncer qu'on va la passer sous silence ». Cette figure porte également les noms de paralipse et d'antiphrase. La Rhétorique à Herennius en donne la description suivante :

Il y a prétérition quand nous affirmons laisser de côté ou ignorer ou ne pas vouloir dire ce que nous sommes précisément en train de dire. Exemple: "En effet je parlerais de vos jeunes années que vous avez livrées au bon plaisir de tous si je pensais le moment propice. Mais à dessein je n'en parle pas. Je ne dis pas non plus que les tribuns vous ont reproché vos absences aux armées. [...] De tout cela je ne dis rien. Je reviens à ce qui est l'objet du procès » (IV, 37).

J'examinerai d'abord les diverses formes de la prétérition dans le discours. J'analyserai ensuite les effets de leur utilisation selon les différentes modalités sous lesquelles elle se présente. Enfin, j'envisagerai les possibilités d'ajustement stratégique potentielles de ce dispositif rhétorique, et exposerai les formes de dérapage que son utilisation peut occasionner.

La prétérition consiste essentiellement, pour le locuteur, dans le fait d'annoncer explicitement qu'il va faire abstraction d'une chose tout en la mentionnant ${ }^{3}$. L'énonciateur fait comprendre son intention de ne pas livrer une certaine information, et, ce faisant, la transmet quand même. C'est pourquoi cette figure est parfois appelée "fausse réticence ». Pour mieux saisir les manifestations possibles de la prétérition, je tenterai de répondre aux deux questions suivantes : 1) Par quels moyens l'énonciateur peut-il faire comprendre qu'il ne va pas transmettre une certaine information? et 2) Comment fait-il pour la transmettre quand-même? 


\section{Comment fait-on comprendre que l'on ne va pas dire quelque chose}

7 La première façon pour un énonciateur de faire comprendre qu'il ne va pas parler de quelque chose ou dire quelque chose est de l'annoncer explicitement. L'exemple (1) utilise une approche directe de ce type :

(1) Je ne vous raconterai pas comment ma mère préparait le café pour mon père tous les matins.

(www.stanfordspokenword.com/poems)

(2) "Je ne dis pas que le pape est ignorant ", dit Yomakogullari, «mais il a dit des choses si abominables sur l'Islam qu'on ne peut faire autrement que de conclure qu'il ne connaît rien à notre religion. L'Islam signifie la paix ».

(NRC Handelsblad, 28 novembre 2006)

Il existe également des façons plus indirectes de faire comprendre que l'on ne va pas dire quelque chose ni en parler. Un énonciateur peut, par exemple, mettre en valeur le fait que l'une ou plusieurs des conditions de félicité assurant la réussite d'un acte de langage ne sont pas remplies, impliquant par là que la réalisation de cet acte n'est pas possible.

Les conditions de réussite d'un acte de langage assertif sont les suivantes ${ }^{4}:$ il faut que :

- L croie que la proposition exprimée dans l'assertion est vraie et correcte (condition de sincérité)

- L croie qu'il peut avancer des preuves à l'appui de la proposition exprimée (condition préparatoire 1)

- L croie que l'information contenue dans la proposition est pertinente pour le récepteur (nouvelle importante etc.) (condition préparatoire 2)

- L croie qu'il a le droit ou qu'il peut s'engager quant à la justesse de la proposition (c'est-à-dire qu'il n'existe aucune raison d'ordre moral, légal ou pratique, ni de conventions sociales qui l'empêchent de le faire) (condition préparatoire 3).

10 En contestant la réalisation de l'une des conditions de réussite d'un acte de langage assertif spécifique, le locuteur peut faire comprendre indirectement qu'il n'est pas disposé à l'accomplir. Je donnerai un exemple pour chacune des quatre conditions de réussite citées plus haut.

Négation de la réalisation de la condition de sincérité :

(3) Bush [...] met le Congrès en garde contre la limitation du financement de la guerre [...] : «Je ne crois pas qu'une personne qui n'est pas d'accord avec mon point de vue soit un mauvais patriote. D'un autre côté, je pense qu'il est important que le peuple comprenne les conséquences du fait de ne pas donner à nos troupes les ressources nécessaires pour faire leur travail ».

(www.usatoday.com/news/ washington/2007-02-26-democrats-iraq)

Négation de la réalisation de la condition préparatoire 1 :

(4) Je n'ai aucune raison d'accuser le CERT de faire ceci de façon systématique, mais il est certain que l'utilisation qu'il a faite de l'article de Stanford soulève certaines questions.

(www.freedom-to-tinker.com)

13 Négation de la réalisation de la question préparatoire 2 :

(5) Pour des raisons valables, l'Institut Daisy a choisi d'être bref dans cette page. Pour le moment, il n'est pas important d'entrer dans les détails de la façon dont cette organisation en est venue à communiquer avec ces Ambassadeurs cosmiques 
de splendeur, de générosité et de bonne volonté, progressistes, compatissants et possédant des dons d'un autre monde, en particulier dans ce cas, et il n'est pas nécessaire de s'étendre sur la façon dont ils transmettent d'amples prédictions à court terme en matière de découvertes et autres domaines, qui surprennent régulièrement même les esprits les plus sophistiqués ou les plus simples.

(www.daisyinstitute.com/predictions.htm)

Négation de la réalisation de la condition préparatoire 3 :

(6) Il me semblerait inconvenant de me vanter de leurs notes, aussi je vous dirai simplement que personne n'a jamais ramené à la maison aucune consonne.

(bonald3. blogspot.com/2006_03_01_archive.html)

(7) Dans le silence de la nuit un son étrange me parvint ; si j'avais été un peu plus sobre, j'aurais pu vous dire que c'était le bruit d'une foule.

(www.demolitionmag.com/demolitionporter.htm)

\section{Techniques pour masquer la contradiction inhérente à la prétérition}

Comme nous l'avons vu, la caractéristique de la prétérition consiste dans le fait, pour l'énonciateur, d'annoncer qu'il va passer une chose sous silence, tout en la mentionnant tout de même. Quelles techniques emploie-t-il pour le faire sans trop attirer l'attention sur cette contradiction?

Pour répondre à cette question, il me semble nécessaire d'opérer une distinction entre les diverses formes de la prétérition. Il existe d'une part des cas de prétérition dans lesquels le locuteur prend ses distances par rapport à la proposition, et d'autre part des cas où il nie qu'il va dire, mentionner ou parler de quelque chose. Lorsque le locuteur affirme qu'il n'est pas en train de dire quelque chose (exemple 2), ou qu'il nie la réalisation de la condition de sincérité (" je ne crois pas que », exemple 3), ou celle de la condition préparatoire impliquant qu'il possède la preuve de son affirmation (exemple 4), il indique par là qu'il n'est pas disposé à adhérer à une certaine proposition ${ }^{5}$. Le cas où le locuteur annonce qu'il ne va pas dire quelque chose (exemple 1), ou déclare que les deuxième et troisième conditions préparatoires ne sont pas remplies (exemples 5 à 7) est différent. Il ne récuse pas alors le fait qu'il soit disposé à adhérer à la proposition, mais seulement qu'il soit prêt à parler de quelque chose (ou capable de le faire). Le fait qu'il souscrive à la ou aux propositions qu'il n'est pas disposé à exprimer reste alors implicite ou présenté comme évident. Prenez notamment l'exemple 6: le locuteur déclare qu'il ne peut pas affirmer qu'il a bien entendu le bruit d'une foule, parce qu'il était trop saoul. Il ne nie pas cependant son adhésion au fait qu'il s'agissait bien du bruit d'une foule.

17 Cette différence a des conséquences sur les moyens utilisés par le locuteur pour éviter d'être ouvertement incohérent lorsqu'il annonce qu'il ne va pas dire quelque chose tout en le faisant. Si l'argumentateur ne faisait aucun effort pour masquer la contradiction inhérente à la prétérition, il produirait des phrases du type :

(8) je ne dis pas qu'il est malade, mais il est malade [non p, mais p]

(9) Je ne vais pas vous dire quelle était sa maladie, qui était la grippe

[Je ne vais pas accomplir l'acte de langage $\mathrm{A}$, que je suis en train d'accomplir]

Il est probable que des formes d'incohérence si flagrantes desserviraient le locuteur dans sa tentative de faire accepter son point de vue par l'autre partie. Comment procède-t-il pour masquer ce type d'incohérence? 


\section{information au récepteur, diverses techniques peuvent être employées pour camoufler} la contradiction. Une des façons d'obtenir cet effet consiste à éviter d'utiliser une proposition du type tout-ou-rien, comme de dire, par exemple qu'on ne va pas parler de quelque chose, et d'utiliser à la place une formulation plus nuancée comme «Je ne vais pas entrer dans les détails" ou "Je ne vais pas m'étendre sur ». Les critères permettant de juger si un propos est détaillé ou non étant dans une large mesure relatifs et subjectifs, il est difficile d'accuser quelqu'un de trop entrer dans les détails quand il prétend lui-même ne pas le faire. Cette technique est employée dans l'exemple 10 :

(10) Je ne vais pas entrer dans les détails de ma dispute en public avec le gardien de parking qui a garé ma voiture et volé toutes les pièces de vingt-cinq cents qui étaient dans le cendrier, et a dit quelque chose dans le genre : « Non, je n'ai pas pris vos pièces de vingt-cinq cents, il n'y en avait pas quand vous m'avez laissé la voiture ", auquel j'ai répondu à peu près: « Ouais, bien sûr, ce que vous ne savez pas, c'est que j'ai vérifié avant de vous la laisser et qu'il y avait près de deux dollars en pièces de vingt-cinq cents qui n'y sont plus maintenant et je me demande bien qui les a prises »; il a rétorqué quelque chose comme "Vous êtes malade et je déteste votre bagnole, alors tirez-vous et ne revenez jamais ", et quand il s'est éloigné, une poignée de pièces de vingt-cinq cents est tombée de sa poche.

(www.pauldavidson.net/2004/03/11/today-i-have-nothing-to-say/) ${ }^{6}$

Il existe une deuxième possibilité qui consiste à utiliser une construction du type " Je ne vais pas vous dire comment », comme dans l'exemple 11:

(11) Je ne vous dirais pas comment l'avion a plongé et s'est enfoncé dans les nuages pendant presque une heure durant laquelle je suis resté à gémir sur mon siège, complètement dégrisé, me demandant à quoi ressemblaient les cieux.

(www.knotmag.com/?article $=532-27 k$ )

Cette façon d'introduire l'information peut être efficace en raison de l'ambiguïté potentielle de la conjonction de subordination « comment », qui peut se référer soit à la façon dont on fait quelque chose, soit introduire un état de fait. Pour prendre un exemple, une phrase comme :

(12) Je ne vous dirais pas comment j'ai perdu tout mon argent au Casino

peut être comprise de deux façons, (12a) et (12b) :

(12a) Je ne vous dirais pas de quelle façon exactement j'ai perdu tout mon argent au Casino.

(12b) Je ne vous dirais pas que j'ai perdu tout mon argent au Casino.

Le second cas est clairement une prétérition du type auto-échec, puisque l'information que le locuteur déclare ne pas donner est exactement celle qu'il fait passer. Mais l'ambiguïté de la construction avec « comment " peut faire passer ce fait inaperçu. Si l'énonciataire interprète la construction comme l'annonce du fait que l'autre partie ne va pas décrire en détails la façon dont quelque chose est arrivé, il peut laisser passer le 
fait que l'énonciateur ait bien transmis l'information qu'il a affirmé ne pas avoir l'intention de donner.

La troisième façon de réaliser une prétérition sans être ouvertement incohérent est d'utiliser une construction qui pourrait également convenir dans le cas d'une véritable omission, comme : « Nous ne parlerons pas de X aujourd'hui », où X est un nom ou une phrase, et pas une proposition entière. L'exemple 13 est une véritable omission :

(13) Je n'aborderais ici pas le sujet de la procédure de sélection, car je pense qu'il vaut mieux en discuter pendant la réunion du personnel.

Si la même construction est utilisée avec un nom ou une expression nominale possédant une connotation négative ou positive, elle peut cependant être employée pour transmettre exactement l'information qu'on veut transmettre tout en prétendant ne pas le faire, comme dans l'exemple $14^{7}$ :

(14) Nous n'aborderons pas ici le sujet du déficit budgétaire.

(http://www.virtualsalt.com/rhetoric.htm)

Ce type de construction présente également un autre avantage : l'information (c'est-àdire l'existence du déficit budgétaire) est introduite comme s'il s'agissait déjà d'un fait admis, qui relève du consensus, et ne requiert donc aucune attention critique ${ }^{8}$.

La quatrième façon pour un énonciateur de faire une affirmation ou de porter une accusation tout en déclarant ne pas le faire et sans paraitre incohérent est d'utiliser le conditionnel passé. Celui-ci implique que la non-réalisation d'une condition spécifique empêche le locuteur de faire une affirmation. Il le fait cependant, comme dans l'exemple 15, mais celle-ci ne semble alors plus être adressée à la personne en question, ce qui permet ici au locuteur de nier avoir traité son ami de menteur.

(15) Si tu n'étais pas mon ami, je dirais que tu es un menteur.

\section{Effets potentiels de la prétérition}

Selon la Rhétorique à Herennius, l'un des emplois importants de la prétérition consiste à faire passer une information ou une preuve sans attirer l'attention sur le fait qu'on est en train de le faire :

Cette figure est utile s'il est bon d'indiquer à mots couverts un fait qu'il n'y a pas lieu d'exposer publiquement, si l'affaire est trop longue ou pas assez connue, si l'on ne peut la raconter clairement ou si elle peut être si aisément contestée qu'il est préférable de la suggérer par prétérition plutôt que de développer un discours qui serait contredit (IV, 37).

Usher donne une définition similaire de cette figure de pensée: "elle est utilisée comme un moyen de présenter une preuve de façon telle que celle-ci soit acceptée par l'auditoire même si sa véracité ou sa valeur sont douteuses » (1965 : 175). Il donne la description suivante pour illustrer le fonctionnement de la prétérition :

L'Occultatio [préterition] est donc utilisée pour présenter des données qui, si elles étaient examinées de façon critique par un jury vigilant, seraient considérées comme fausses ou réfutables. Elle permet à l'orateur d'avancer une affirmation qui sera mentalement prise en note par le jury en sa faveur, sans qu'il ne l'examine cependant de trop près, puisqu'il semble lui-même la considérer comme inutile ou hors propos par rapport à son argument. Par ce moyen il est donc possible d'introduire avec aplomb des arguments faibles ou une fausse preuve, en feignant de les omettre (176). 

particulier des arguments sans que ceux-ci ne fassent l'objet d'une attention extrême, mais en atteignant cependant l'auditoire. D'autres auteurs, néanmoins, considèrent la prétérition comme une façon de mettre en relief les données prétendument omises. Lanham, par exemple, la définit comme une manière de "mettre une chose en relief en la passant sous silence d'une façon significative» (1991: 104). Et Dupriez distingue entre «les semi-prétéritions qui mettent à peine l'accent sur l'énoncé » et «la vraie prétérition» qui «est une forme de pseudo-simulation, dissimulant pour mieux révéler » (1991: 354).

32 Je pense, pour ma part, que la prétérition peut être une combinaison de mise en valeur et de dissimulation. L'énonciateur qui utilise une prétérition ne se contente pas de passer quelque chose sous silence ou de s'abstenir de réaliser un acte de langage spécifique, il nie explicitement adhérer à une certaine proposition ou annonce clairement qu'il ne va pas parler de quelque chose. En niant son adhésion à une proposition, il rend celle-ci plus significative aux yeux de son auditoire. Comme le fait observer Clarck (1975) au sujet de l'affirmation célèbre de Richard Nixon «Je ne suis pas un escroc », la dénégation présuppose que l'auditoire croie ou soit susceptible de croire ce qui est nié9. Et lorsque le locuteur annonce qu'il ne va pas aborder un certain sujet, il attire également par là l'attention sur l'acte de langage qu'il affirme ne pas vouloir réaliser, puisqu'il le fait en principe uniquement dans une situation où l'auditoire s'attend à ce qu'il réalise un certain acte de langage, ou bien où il aurait luimême voulu le faire, mais s'en abstient néanmoins. Pour cette raison, nier que l'on va réaliser un certain acte de langage focalise l'attention de l'auditoire sur le fait qu'on aurait pu ou qu'on aurait souhaité le faire.

Nier son adhésion à un certain acte de langage ou récuser le fait qu'on va l'accomplir attire donc d'ores et déjà l'attention de l'auditoire sur l'information qu'on déclare ne pas fournir. Dans la suite, cette information est néanmoins donnée, mais elle est généralement présentée de façon à camoufler la contradiction entre ce que le locuteur déclare être en train de faire et ce qu'il fait en réalité. Ainsi la prétérition, lorsqu'elle est combinée avec des techniques de présentation spécifiques, peut-elle être considérée comme une forme à la fois de mise en valeur et de dissimulation.

\section{Prétérition et ajustement stratégique}

Je souhaiterais à présent traiter du rôle de la prétérition dans le cadre du processus d'ajustement stratégique employé par l'argumentateur. Selon Eemeren et Houtlosser, chacune des étapes dialectiques selon lesquelles se déroule la discussion critique possède son équivalent rhétorique. On peut donc distinguer pour chacune d'entre elles des objectifs rhétoriques et dialectiques (2002 : 138). Jusqu'à présent, nous avons décrit la prétérition principalement comme une technique efficace pour présenter des arguments de façon à ce qu'ils soulèvent le moins de critiques possibles, c'est-à-dire en la considérant comme un mécanisme qui peut être employé au stade argumentatif de l'échange de vue. A mon point de vue, la prétérition peut également être utilisée au cours des autres étapes de la discussion.

35 Au stade de la confrontation, le but dialectique des participants étant de faire ressortir le désaccord, leur objectif rhétorique sera de définir celui-ci de la façon la plus

Argumentation et Analyse du Discours, 2 | 2009 
avantageuse pour soutenir leur point de vue (van Eemeren et Houtlosser $2002: 138$ ). Par exemple, ils présenteront leur opinion de manière à la rendre moins accessible à la réfutation, comme dans le cas 16 . Ou bien ils tenteront de critiquer la position de l'autre partie en faisant en sorte qu'on ne puisse leur demander des comptes, comme dans l'exemple 17.

Présenter un point de vue sous forme de prétérition:

(16) «Je ne dis pas que le pape est ignorant » dit Yomakogullari, «mais il a dit des choses si abominables sur l'Islam qu'on ne peut faire autrement que de conclure qu'il ne connaît rien à notre religion. L'Islam signifie la paix ».

(NRC Handelsblad, November 28, 2006)

Critiquer le point de vue de l'autre partie au moyen d'une prétérition :

(17) Dan :J'ai vu l'émission de sketchs « the Big Bite » à la télévision hier soir. Quelle médiocrité. Quel manque d'originalité.

Joe M. : Oh, Dan, il serait trop facile de dire « faites mieux » mais il y a ici des gens qui gagnent leur vie en aidant des comédiens à se faire connaître. Je préfère regarder et encourager une comédie locale pour des téléspectateurs locaux que n'importe quelle émission de télé-réalité importée.

(phorums.com.au/archive/index.php/t-33805.html - 12k)

Lors de la phase d'ouverture, où le but dialectique est d'établir une base de départ claire pour la discussion en s'accordant sur les données d'origine, l'objectif rhétorique de chaque partie sera de formuler le point de départ qui sert le mieux son propre intérêt. Les locuteurs pourront, entre autres, proposer une base de discussion à l'autre partie, ou lui en attribuer une ${ }^{10}$. Dans l'exemple 18, le locuteur affirme qu'une certaine donnée a déjà été acceptée comme point de départ par l'emploi d'une prétérition :

(18) Pour moi, le mode de vie baby boomer consiste à exploiter au maximum les possibilités qui s'offrent à nous aujourd'hui, en particulier celle de travailler de la maison. Je n'ai pas besoin de vous rappeler que nous l'avons déjà fait. Lorsqu'on trouve une opportunité et qu'elle semble bonne, on l'adopte. C'est précisément une des choses que nous pouvons choisir de faire. Et, tout comme vous, je préfèrerais le faire dans la facilité que dans la difficulté [...]. Joignez-vous au nombre des baby boomer. Travaillez de chez vous.

(ezinearticles.com/?Baby-Boomers---Work-From-Home-Is-An-Option\&id=841077 $40 \mathrm{k}-)$

Lors de l'étape argumentative, le but dialectique est d'avancer une argumentation et de critiquer celle de l'autre. L'objectif rhétorique de cette phase est «de construire l'argumentation la plus forte et de lancer l'offensive la plus efficace » (van Eemeren \& Houtlosser 2002 : 139). Pour ce faire, les argumentateurs vont, entre autres, tenter de présenter leurs arguments de façon à ce qu'ils semblent les plus forts possible. Dans l'exemple 19, un des arguments en faveur de la position selon laquelle « les Américains doivent avoir plus de vacances» est présenté sous la forme d'une prétérition. Le locuteur suggère qu'il aurait pu avancer un argument plus fort que celui qu'il a fourni, mais qu'il n'a pas eu besoin de le faire, car même sans cet argument supplémentaire, son argumentation est déjà suffisamment forte.

Présenter un argument sous la forme d'une prétérition :

(19) Ah, pour poursuivre mes perpétuelles fanfaronnades au sujet du travail en général... Saviez-vous que les travailleurs chinois ont une moyenne de trois semaines de vacances par an ??? J'ai lu ça dans un article la semaine dernière, je n'en revenais pas. Ils n'ont pas la liberté de parole, mais ils ont plus de vacances que nous ? [...] Je pense vraiment que nous méritons mieux. Le «meilleur » pays du monde ne peut pas donner à ses employés autant de vacances qu'en Chine? Je ne 
mentionne même pas la quantité de jours de vacances dont bénéficient les Européens.

(tjparker.spaces.live.com/blog/cns!7BF842B4D45B5A92!1538.entry-44k-)

41 Le but dialectique de la phase de conclusion est la détermination des résultats de la discussion : le proposant peut-il maintenir son point de vue ou l'opposant ses réserves. L'objectif rhétorique de chaque partie est de "proclamer la victoire » (van Eemeren \& Houtlosser 2002: 139). Dans l'exemple 20, l'opposant termine la discussion en maintenant sa position de doute, mais le fait sous la forme d'une prétérition, probablement pour faciliter la clôture de la discussion en sa faveur sans plus de cérémonie, puisque la « promesse » donne l'impression qu'il est positif (ou du moins le ferait-elle, sans l'ajout du « houp !»).

Maintenir le doute grâce à la prétérition :

(20) Merci beaucoup, et je promets de ne pas mentionner le fait que je ne suis pas d'accord (houp!).

(www.physicsforums.com/showthread.php?t=237\&page=13-117k-)

J'espère que ces exemples suffiront à faire comprendre que le dispositif stylistique de la prétérition peut être appliqué par les argumentateurs à tous les stades de la discussion argumentative, et être analysé comme contribuant au processus d'ajustement stratégique que ceux-ci mettent en œuvre. Bien que l'utilisation de la prétérition ne débouche pas nécessairement sur un discours fallacieux, elle présente un véritable risque de dérapage du processus. Elle peut permettre une fuite caractérisée devant la charge de la preuve, comme dans l'exemple 16, où l'argumentateur prévient la critique en déclarant que la position qu'il défend n'est en fait pas celle qu'il veut défendre. De façon plus générale, l'utilisation de la prétérition peut constituer un moyen de violer impunément les règles de la discussion critique. Dans l'exemple 17, par exemple, l'argumentateur commet en fait un paralogisme ad hominem du type tu quoque lorsqu'il réagit de façon critique au point de vue de l'autre partie par sa remarque "Faites mieux ", mais, en introduisant sa réaction par une prétérition, il échappe à la responsabilité de ce paralogisme. Technique permettant de dissocier un niveau " officiel» et un plan "officieux» dans le discours, la prétérition est, d'une façon générale, un moyen, pour le locuteur, d'éviter de prendre l'entière la responsabilité de ses propos.

\section{BIBLIOGRAPHIE}

Clark, Herbert H. 1988. Bridging. Theoretical Issues in Natural Language Processing. Proceedings of the 1975 workshop on Theoretical issues in natural language processing. Collins Cobuild English Language Dictionary. (London and Glasgow : Collins)

Ducrot, Oswald. 1984. Le dire et le dit (Paris : Minuit)

Dupriez, Bernard. 1991. A Dictionary of Literary Devices. Translated and adapted by Allbert W. Halsall (New York, London etc. : Harvester Wheatsheaf) 
Eemeren, Frans H. van \& Peter Houtlosser. 2002. « Strategic maneuvering: maintaining a delicate balance », Eemeren \& Houtlosser (eds). Dialectic and Rhetoric: The Warp and Woof of Argumentation Analysis (Dordrecht, Boston and London : Kluwer Academic Publishers), 119-130

Eemeren, Frans H. van, Peter Houtlosser \& Francisca Snoeck Henkemans. 2007. Argumentative Indicators in Discourse. A Pragma-Dialectical Study (Dordrecht : Springer)

Lanham, Richard A. 1991. A Handlist of Rhetorical Terms. $2^{\text {nd }}$ edition (Berkeley, Los Angeles \& Oxford : University of California Press)

Levene, D. S. 2004. «Reading Cicero's narratives », Powell, J. \& J. Paterson (eds). Cicero the Advocate, (Oxford : Oxford University Press), 117-146

Perelman, Chaim \& Lucie Olbrechts-Tyteca. 1969. The New Rhetoric. A Treatise on Argumentation. J. Wilkinson \& P. Weaver, Transl. (Notre Dame and London : University of Notre Dame Press)

Rhétorique à Herennius 1989. Ed. Guy Achard (Paris : Les Belles Lettres).

Searle, John R. \& Daniel Vanderveken (1985). Foundations of Illocutionary Logic (Cambridge : Cambridge University Press)

Schmid, Hans-Jörg. 2001. « Presupposition can be a bluff : How abstract nouns can be used as presupposition triggers », Journal of Pragmatics 33, 1529-1552

Snoeck Henkemans, Francisca. 2005. "What's in a name? The use of the stylistic device metonymy as a strategic manoeuvre in the confrontation and argumentation stages of a discussion ", Proceedings Conference The Uses of Argument (Hamilton : Ontario Society for the Study of Argumentation), 433-441

Snoeck Henkemans, Francisca. 2007. « Manoeuvering strategically with rhetorical questions », Proceedings of the 6th conference of the international society for the study of argumentation (Amsterdam : Sic Sat.), 1309-1313

Usher, Stephen. 1965. « Occultatio in Cicero's speeches », The American Journal of Philology. Vol. 85, 342, 175-192

\section{NOTES}

1. Ces trois aspects de l'ajustement stratégique sont comparables, dans une large mesure, avec les notions de « choix », de « communion » et de «présence » chez Perelman et Olbrechts-Tyteca (1969).

2. Deux exemples de ce type de recherche se trouvent dans Snoeck Henkemans 2005 et 2007.

3. La réticence (ou aposiopèse ou apophasie) est le terme utilisé pour désigner une véritable omission, dans laquelle le locuteur réalise son intention déclarée de passer quelque chose sous silence (Usher 1969 : 177). Selon Usher, la réticence « peut créer un effet rhétorique en suggérant l'existence d'un nombre important de preuves pertinentes sur lesquelles le locuteur n'a pas l'intention d'attirer l'attention » (Usher 1969 : 177).

4. Mon analyse des conditions de réussite des assertions se fonde sur la description des catégories de base des assertions de Peter Houtlosser (1995 : 103-106), elle-même basée sur les conditions de félicité formulées par Searle et Vanderveken (1985).

5. Cette dernière condition constitue, avec la condition de sincérité, une sous-maxime de la maxime de qualité de Grice. En indiquant que l'une de ces conditions n'est pas remplie, le locuteur peut donc faire comprendre que son affirmation n'est peut-être pas sincère, et qu'il ne peut par conséquent pas être tenu pour engagé par la proposition qui y est exprimée. 
6. Cet exemple constitue en fait un cas extrême en raison de la quantité de détails fournis. Ce cas extrême de prétérition dans lequel tous les détails qu'on déclare vouloir omettre sont donnés est parfois appelé prolipse.

7. Comme le signale Levene, «l'effet de la praeteritio dans une narration différera selon qu'elle constitue un simple rappel de quelque chose qui a déjà été exposé en détail, ou qu'elle est la seule source d'information offerte aux allocutaires » (2004:135).

8. Schmid (2001) discute de constructions comparables avec un nom abstrait comme « Le fait est que ", ou "Le problème est que ", par lesquels l'énonciateur peut manipuler son énonciataire. Les présuppositions sont souvent, selon lui, des bluffs par lesquels le locuteur « fait croire à tort à son récepteur que certaines informations ne requièrent pas d'attention ni même de réflexion particulières, car elles représentent des certitudes familières et mutuellement partagées » (1548). 9. Une analyse similaire est faite par Ducrot (1984: 216-217), qui déclare que les phrases contenant une négation (polémique) impliquent un dialogue avec une second "voix" (silencieuse) soutenant le point de vue opposé.

10. Dans van Eemeren, Houtlosser and Snoeck Henkemans (2007: 90-92) est présenté un profil dialectique de la phase d'ouverture, détaillant les différentes façons qu'ont les participants à une discussion critique de proposer un point de départ ou de répondre à une telle proposition. Les divers indicateurs de ces stratégies à cette étape de la discussion sont également débattus.

\section{RÉSUMÉS}

Cet article explore le rôle que peut jouer le procédé stylistique de la praeteritio (ou paralipsis) dans les tentatives effectuées par les argumentateurs pour concilier leurs objectifs rhétoriques et dialectiques par un processus d'ajustement stratégique («strategic manoeuvering »), lorsqu'ils poursuivent une procédure dialectique de résolution des différends. On examine tout d'abord la façon dont la praeteritio peut être réalisée dans le discours. On analyse ensuite les effets que peut avoir son usage en fonction des moyens de présentation employés. Cette analyse permet d'établir les possibilités d'ajustement stratégique qu'offre ce procédé dans les différentes étapes de la discussion argumentative. Finalement, on indique comment les types d'ajustement stratégique dans lesquels la praeteritio est susceptible d'intervenir peuvent déraper, et quelles violations des règles de la discussion critique ces dérapages peuvent provoquer.

In this paper, it is investigated what role the stylistic device of praeteritio (or paralipsis) can play in arguers' attempts to reconcile their rhetorical with their dialectical aims by manoeuvring strategically when carrying out particular discussion moves of the dialectical procedure for resolving a dispute. First, attention will be paid to the ways in which praeteritio can be realized in discourse. Next, an analysis is given of the effects the use of praeteritio may have due to the presentational means that are employed. This analysis will be used to establish the possibilities for strategic manoeuvring with this device in the different stages of an argumentative discussion. Finally, an indication is given of how the types of strategic manoeuvring that a praeteritio can be instrumental in may derail, and in which violations of the rules for critical discussion such derailed manoeuvrings may result. 
INDEX

Keywords : burden of proof, paralipsis, praeteritio, reticence, strategic manoeuvring

Mots-clés : ajustement stratégique, charge de la preuve, paralipsis, praeteritio, réticence

\section{AUTEURS}

\section{FRANCISCA SNOECK HENKEMANS}

Université d'Amsterdam 